\section{Cureus}

Received 09/20/2014

Review began 09/22/2014

Review ended 10/08/2014

Published 10/09/2014

\section{Copyright 2014}

Doherty et al. This is an open access article distributed under the terms of the Creative Commons Attribution License CC-BY 3.0., which permits unrestricted use, distribution, and reproduction in any medium, provided the original author and source are credited.

\title{
Using Simulated Transport Calls to Identify Diversity of Knowledge and Care Plans among the Multidisciplinary Team
}

\author{
Elizabeth Doherty ${ }^{1}$, Christine Rachwal ${ }^{1}$, Kristen Lindamood ${ }^{1}$, Caitlin O'Brien ${ }^{1}$, Peter \\ Weinstock ${ }^{2}$ \\ 1. Newborn Medicine, Boston Children's Hospital 2. Anesthesia and Critical Care Medicine, Boston \\ Children's Hospital
}

$\square$ Corresponding author: Elizabeth Doherty, elizabeth.doherty@childrens.harvard.edu Disclosures can be found in Additional Information at the end of the article

\section{Abstract}

Research has shown that patient safety and quality of care provided is affected by the knowledge, technical ability, and behavioral skills of each member of the multidisciplinary care team. All NICU patients at Boston Children's Hospital (BCH) are born at outside facilities. Thus, transport call preparedness is fundamental to providing exceptional care for NICU patients.

Our study aims to answer the question: Does significant variance exist among the multidisciplinary team members in the interpretation of transport call data and the subsequent preparation that occurs when caring for critically ill neonates?

The 2011 NICU Crisis Resource Management (CRM) course reflects results from a multidisciplinary needs assessment. Three transport calls for neonate with persistent pulmonary hypertension of the newborn (PPHN) were received by a multidisciplinary team. Each member completed a survey following each call. After the final transport call, the patient arrived in the simulator suite and the course progressed.

One hundred forty interdisciplinary participants completed the course. After listening to the transport calls, $85.5 \%$ of participants categorized the patient as category, RED (Emergent/Urgent resuscitation-high acute status), while $14.5 \%$ of participants selected category, YELLOW (Patient in clinical transition or requiring time sensitive procedures). Of the healthcare providers, the highest percentage group selecting category YELLOW was the fellow group (18\%), while 0\% of the NNP group selected YELLOW. Blood pressure mean goal recommendations were more varied among the groups. Eighty-five per cent of attendings, 75\% of neonatal nurse practitioners (NNPs), $75 \%$ of fellows, and $55 \%$ of RNs selected $40-50 \mathrm{mmHg}$; while $15 \%$ of attendings, $25 \%$ of NNPs, $12.5 \%$ of fellows, and $33 \%$ of RNs selected $50 \mathrm{mmHg}$ or more. Importantly, $100 \%$ of participants, including attendings, NNPs, respiratory therapists (RTs), fellows, and RNs, said they would have a team "huddle" prior to the arrival of this infant.

Within the variance that exists in regard to knowledge, experience, and practice among the multidisciplinary groups, attendings and NNPs tended to be more aligned with their assessment and approach. Simulated transport calls provide a safe environment to uncover these differences and to allow for future study to improve teamwork and to provide the best care for the critically ill neonate.

Categories: Medical Simulation, Pediatrics

Keywords: neonatology, simulation, teamwork

How to cite this article

Doherty E, Rachwal C, Lindamood K, et al. (October 09, 2014) Using Simulated Transport Calls to Identify Diversity of Knowledge and Care Plans among the Multidisciplinary Team. Cureus 6(10): e217. DOI 10.7759/cureus. 217 


\section{Introduction}

Patients in the neonatal intensive care unit (NICU) are considered among the most vulnerable. An immature immune system, an inability to communicate, a prolonged hospital course, and numerous multidisciplinary health care providers all place the neonate's safety at risk. Studies have shown that $75 \%$ of these hospitalized infants will have an adverse event during their hospitalization with an inversely proportionate increase with lower gestational age [1-2].

Research has shown that the safety of these patients and the care they receive is affected by the knowledge, technical ability, and behavioral skills of each member of the multidisciplinary care team [3]. As is common for all multidisciplinary teams, each member has a diverse background of training and experiential knowledge that is utilized when caring for the neonatal population. The expectation of these health care professionals is to be highly educated in their discipline with an excellent fund of knowledge and skill set within which they practice. Each of these providers must also rely on accurate information and assessments transmitted among members of the care team. This information must be delivered in a concise and timely fashion so care is not interrupted. Safe, efficient, and effective patient care can only be accomplished through collaboration among the caregivers as team members. However, despite our expectations, errors occur in judgment, communication, and practice.

All NICU patients at BCH are born at outside facilities, and nearly all infants are admitted via emergency medical transport. Thus, transport call preparedness is fundamental to providing exceptional care for NICU patients. In our study, we were interested in answering the question: Does significant variance exist among the multidisciplinary team members in the interpretation of transport call data and the subsequent preparation that occurs when caring for critically ill neonates? Here, we report the results of a two-year simulation curriculum focused on the multidisciplinary care of a critically ill neonate with persistent pulmonary hypertension of the newborn (PPHN).

PPHN is a major clinical problem in the NICU and is estimated to occur in two per 1000 term infants. Studies reveal that some degree of PPHN complicates the course of more than $10 \%$ of all neonates with respiratory failure. With current medical therapy, the mortality for PPHN remains between $5-10 \%$, and approximately $25 \%$ of all infants with moderate to severe PPHN will exhibit significant neurodevelopmental impairment at 12-24 months [4-7]. The NICU Crisis Resource Management (CRM) 2011 curriculum was based on a multidisciplinary NICU Staff Needs Assessment where staff recognized variance and frustration in practice management for patients with PPHN. In the simulation course, the care of the critically ill infant begins with the first phone call to $\mathrm{BCH}$ from the physician caring for the infant at the outside hospital.

\section{Materials And Methods}

We utilized the BCHSP on-site simulation suite to provide the realistic NICU setting for our multidisciplinary training program in CRM. Care of the infants in the NICU at BCH is managed by a multidisciplinary team of nurses, respiratory therapists (RTs), neonatology fellows, neonatal nurse practitioners (NNPs), and neonatology attending physicians. The monthly NICU CRM courses are a mandatory requirement for multidisciplinary training in the NICU. Attendance at the monthly CRM course includes five to eight nurses, an RT, a neonatal fellow, an NNP, and an attending neonatologist. Medical students frequently observe.

The 2011-2013 NICU CRM course was designed as a seminar program for graduate education that provided:

1. Didactic with review of CRM principles, 


\section{Cureus}

2. Video demonstration of scenarios for participants to review CRM principles, recognizing both appropriate technical and behavioral team training and areas for improvement,

\section{Simulation scenario and debriefing designed as follows:}

a. Three transport calls regarding a critically ill neonate were pre-recorded to be received simultaneously by the multidisciplinary team (RN; NNP; RT; fellow; attending). Each member completed a survey following each transport call (12 questions in total). The complete survey is available on request (elizabeth.doherty@childrens.harvard.edu). Survey questions were designed to assess alignment of answers within and between multidisciplinary groups; not for interpretation of "right or wrong" answers. Participants were first asked to assign a severity score/rating as described by RED (Emergent/Urgent resuscitation-high acute status), YELLOW (Patient in clinical transition or requiring time sensitive procedures), or GREEN (General NICU patient with ongoing ICU needs). Subsequent survey questions focused on three arms of management, including Respiratory/ Ventilator management, Cardiovascular/ BP management, and Neurologic/Sedation. The only identifiers collected were role and years of experience (Table 1).

b. After the final transport call, the patient arrived in the simulator suite and the remainder of the course progressed.

$\begin{array}{lllll}\text { Number of years in your role } & & & \\ \text { Answer options } & 1-5 & 6-10 & 10+ & \text { Response count } \\ \text { RN } & 23 & 27 & 35 & 85 \\ \text { NNP } & 2 & 1 & 5 & 18 \\ \text { RT } & 12 & 5 & 1 & 16 \\ \text { Fellow } & 16 & 0 & 0 & 13 \\ \text { Attending } & 7 & 3 & 3 & \text { Totals } \\ \text { Answered question } & & & & 140\end{array}$

\section{TABLE 1: Participant role and years of experience}

\section{Results}

One hundred forty participants completed the course, including 85 nurses, 8 NNPs, 19 RTs, 16 Neonatal-Perinatal Fellows, and 13 Neonatology Attendings. After listening to the transport calls, $85.5 \%$ of participants categorized the patient as category, RED (Emergent/Urgent resuscitation-high acute status), while $14.5 \%$ of participants selected category, YELLOW (Patient in clinical transition or requiring time sensitive procedures) (Table 2). Of the healthcare providers, the highest percentage group selecting category YELLOW was the fellow 


\section{Cureus}

group (18\%), while 0\% of the NNP group selected YELLOW.

GREEN (General NICU patient with ongoing ICU needs but "acutely/stable")

$0.0 \%$

YELLOW (Patient in clinical transition or requiring time sensitive procedures)

RED (Emergent/Urgent resuscitation - highly acute status)

$85.5 \%$

\section{TABLE 2: Categorization of transport calls by participants}

Blood pressure (BP) mean goal recommendations were more varied among the groups. Overall, $59.9 \%$ of participants selected $40-50 \mathrm{mmHg}, 27 \%$ selected $50 \mathrm{mmHg}$ or more, $11.7 \%$ selected $35-40 \mathrm{mmHg}$, while $1.5 \%$ selected $35 \mathrm{mmHg}$. More specifically, $85 \%$ of attendings, $75 \%$ of NNPs, $75 \%$ of fellows, and $55 \%$ of RNs selected $40-50 \mathrm{mmHg}$; while $15 \%$ of attendings, $25 \%$ of NNPs, $12.5 \%$ of fellows, and $33 \%$ of RNs selected $50 \mathrm{mmHg}$ or more. The choice of a second inotropic agent in addition to dopamine was less varied with $80.7 \%$ of participants selecting epinephrine, $14.8 \%$ selecting dobutamine, $3.7 \%$ selecting milrinone, and $0.7 \%$ selected no additional agent (Table 3).

\section{Answer Options}

No Change; continue to titrate Dopamone to effect

Dobutamine

Epinephrine

Milrinone

Answered question

Skipped question

\section{Response Percent}

$0.7 \%$

$14.8 \%$

$80.7 \%$

$3.7 \%$

5
135

\section{Response Count}

1

20

109

5

TABLE 3: Considering the escalation of inotropic support to $20 \mathrm{mcg} / \mathrm{kg} / \mathrm{min}$; if your patient requires additional support what would you chose as the 2 nd agent?

The figures below (Table 4) show participant responses to: What is the most important intervention upon the infant's arrival to the NICU? 


\section{Cureus}

\section{TABLE 4: Participant response for most important intervention upon patient arrival}

Of the participants answering this question, the RNs and the NNPs were most aligned with $60 \%$ and $62.5 \%$, respectively, selecting blood gas.

Survey questions about sedation had less varied responses. Ninety-six per cent of participants recommended discontinuing morphine to change to fentanyl and versed. Overwhelmingly, 98\% of participants would consider paralysis in this infant due to either inability to achieve blood gas parameters within four hours or due to inability to maintain State Behavioral Scale (SBS) of minus two or both. The one question that resulted in 100\% agreement was that all participants would have a "huddle" prior to the infant's arrival.

\section{Discussion}

The optimal approach to PPHN management is unclear [8]. Substantial variation in clinical practice exists between and within institutions and among multidisciplinary groups; yet the overall objective seems to be consistent focusing on restoring the cardiopulmonary adaptation while avoiding lung injury and any detrimental effects on systemic perfusion [9]. The basic treatment goals include:

* Improve alveolar oxygenation

* Minimize pulmonary vasoconstriction

* Maintain systemic blood pressure and perfusion

* Avoid acidosis

* Promote pulmonary vasodilatation (iNO)

* Consider extracorporeal membrane oxygenation (ECMO) support as last resort

The BCH NICU Multidisciplinary Needs Assessment demonstrated the HCP's desire to practice caring for these critically ill patients with PPHN.

The NICU is by nature an intense, busy unit where critically ill infants are cared for by a multidisciplinary group of health care providers (HCPs). The $\mathrm{BCH}$ team preparation and subsequent management of an infant with PPHN begins with the first transport call. The interpretation of data received and succeeding plans vary by multidisciplinary group, some more than others. Our survey questions focused on 3 arms of management:

* Respiratory/Ventilator management 
* Cardiovascular/ BP management

* Neurologic/ Sedation

Minimal variance existed among disciplines for the survey questions about ventilation strategy and anticipation/procedures for pneumothorax. In addition, all participants recognized the importance of team communication prior to the infant's arrival, as $100 \%$ said they would have a "huddle" prior to the infant's admission.

BP management was consistent among attendings, NNPs, and fellows. Overwhelmingly, they selected a goal mean BP of 40-50 mmHg. The responses from the nurses were more variable. Interestingly, $80 \%$ of participants selected epinephrine as a second agent after dopamine to maintain BP. Eighty per cent of this group was comprised of attendings and NNPs.

Optimizing cardiac function, cardiac output, and systemic oxygen transport is essential and is achieved as needed with volume expansion and inotropic agents (dopamine and dobutamine). Hemodynamic support is provided to increase systemic vascular resistance, to reverse shunting, and to minimize hypoxemia and acidosis. Systemic blood pressure is often optimized at the upper limits or normal. Hypovolemia should be corrected with infusion of colloid-based volume expanders, such as blood. Inotropic agents are widely used for persistent hypotension despite volume expansion. Epinephrine and milrinone are two additional agents are often utilized for BP control in the NICU. Epinephrine is used as clinically indicated and discontinued or titrated based on clinical response. It is unclear why there was a clear preference for epinephrine as a second line agent in this clinical case; perhaps related to practice style or the patient profile in the BCH NICU. Further study could provide more information.

The use of muscle relaxants/paralytics and sedatives is often employed in the repertoire for PPHN management as an aide to facilitate ventilation, to minimize fluctuations in oxygenation, and to provide pain control. The use of paralytics is controversial [10]. Caution should always be used with muscle relaxants to limit continued use to less than 48 hours or to provide daily periods free from muscle relaxation or significant sedation. In our study, 96\% of participants recommended discontinuing morphine to change to fentanyl and versed. Overwhelmingly, 98\% of participants would consider paralysis in this infant due to either inability to achieve blood gas parameters within four hours or due to inability to maintain State Behavioral Scale (SBS) of minus two or both. The group selecting SBS of minus two as the sole reason for paralysis was made up of all nurses and one fellow.

The management of PPHN is widely varied with the ideal combination and doses of therapeutic agents unknown [11]. The results from the preliminary study looking at variance among disciplines in the preparation and management of a critically ill infant with PPHN show a trend toward alignment of attendings and NNPs. Further investigation is needed to uncover the reasons behind choice selections. The variation in practice may be dictated by the lack of standardized guidelines. BCH has implemented a Standardized Clinical Assessment Management Plan (SCAMP) for PPHN. Data collection is underway. The combined efforts of the BCH NICU CRM course and the BCH PPHN SCAMP should help further our knowledge and understanding about the management and teamwork in caring for an infant with PPHN.

\section{Conclusions}

Variance exists in regard to knowledge, experience, and practice among the multidisciplinary groups. The most variance existed in the "arm" of cardiovascular/BP management. Of the groups represented, attendings and NNPs tended to be more aligned with their assessment and approach to interpretation of simulated transport call data and subsequent management in an 
infant with PPHN. Simulated transport calls provide a safe environment to uncover these differences and to allow for future study to improve teamwork and to provide the best care for the critically ill neonate.

\section{Additional Information}

\section{Disclosures}

Human subjects: Consent was obtained by all participants in this study. $\mathrm{BCH}$ issued approval IRB-P00002103 The following protocol was considered exempt from IRB/human subject review on 12/8/2011. Animal subjects: All authors have confirmed that this study did not involve animal subjects or tissue. Conflicts of interest: In compliance with the ICMJE uniform disclosure form, all authors declare the following: Payment/services info: All authors have declared that no financial support was received from any organization for the submitted work. Financial relationships: All authors have declared that they have no financial relationships at present or within the previous three years with any organizations that might have an interest in the submitted work. Other relationships: All authors have declared that there are no other relationships or activities that could appear to have influenced the submitted work.

\section{Acknowledgements}

Denise Casey, RN, MS, CCNP, CCRN (Boston Children's Hospital, Harvard Medical School) Denise.Casey@childrens.harvard.edu Michelle DeGrazia, PhD, RN, NNP-BC (Boston Children's Hospital, Harvard Medical School) Michele.DeGrazia@childrens.harvard.edu Monica Kleinman, MD (Clinical Director, Medical/Surgical Intensive Care Unit; Medical Director, Transport Program, Boston Children's Hospital; Associate Professor of Anesthesia, Harvard Medical School) Monica.Kleinman@childrens.harvard.edu

\section{References}

1. Creating a Culture of Safety in the NICU: the Team STEPPS approach . (2011). Accessed: May 9, 2011: http://www.nicuniversity.org/Education.aspx? ct=CME\%20and\%20CE\%20Lecture\%20Series.

2. Sharek PJ, Horbar JD, Mason W, Bisarya H, Thurm CW, Suresh G, Gray JE, Edwards WH, Goldmann D, Classen D: Adverse events in the neonatal intensive care unit: Development, testing, and findings of an NICU-focused trigger tool to identify harm in North American NICUs. Pediatrics. 2006, 118:1332-40. 10.1542/peds.2006-0565

3. Joint Commission on Accreditation of Healthcare Organizations: The Joint Commission guide to improving staff communication. Joint Commission Resources, Oakbrook Terrace, IL; 2005.

4. Clark RH, Huckaby JL, Kueser TJ, Walker MW, Southgate WM, Perez JA, Roy BJ, Keszler M; Clinical Inhaled Nitric Oxide Research Group: Low-dose nitric oxide therapy for persistent pulmonary hypertension: 1-year follow-up. J Perinatol. 2003, 23:300-3. 10.1038/sj.jp.7210908

5. Neonatal Inhaled Nitric Oxide Study Group: Inhaled nitric oxide in term and near-term infants: neurodevelopmental follow-up of the neonatal inhaled nitric oxide study group (NINOS). J Pediatr. 2000, 136:611-7. 10.1067/mpd.2000.104826

6. Konduri GG, Vohr B, Robertson C, Sokol GM, Solimano A, Singer J, Ehrenkranz RA, Singhal N, Wright LL, Van Meurs K, Stork E, Kirpalani H, Peliowski A, Johnson Y; Neonatal Inhaled Nitric Oxide Study Group: Early inhaled nitric oxide therapy for term and near-term newborn infants with hypoxic respiratory failure: Neurodevelopmental follow-up. J Pediatr. 2007, 150:235-40, 240.e1. 10.1016/j.jpeds.2006.11.065

7. Konduri GG, Solimano A, Sokol GM, Singer J, Ehrenkranz RA, Singhal N, Wright LL, Van Meurs K, Stork E, Kirpalani H, Peliowski A; Neonatal Inhaled Nitric Oxide Study Group: A randomized trial of early versus standard inhaled nitric oxide therapy in term and near-term newborn infants with hypoxic respiratory failure. Pediatrics. 2004, 113:559-64.

8. Walsh-Sukys MC, Tyson JE, Wright LL, Bauer CR, Korones SB, Stevenson DK, Verter J, Stoll BJ, Lemons JA, Papile LA, Shankaran S, Donovan EF, Oh W, Ehrenkranz RA, Fanaroff AA: 


\section{Cureus}

Persistent pulmonary hypertension of the newborn in the era before nitric oxide: practice variation and outcomes. Pediatrics. 2000, 105:14-20.

9. Konduri GG, Kim UO: Advances in the diagnosis and management of persistent pulmonary hypertension of the newborn. Pediatr Clin North Am. 2009, 56:579-600. 10.1016/j.pcl.2009.04.004

10. Park MK: Pediatric Cardiology for Practitioners. Elsevier, Philadelphia; 2007, pp 301.

11. Agrawal A, Agrawal R: Persistent pulmonary hypertension of the newborn: Recent advances in management. Int J Clin Pediatr. 2013, 2:1-11. 10.4021/ijcp79w 